\title{
La surveillance relationnelle de la santé. Du soupçon au soutien : les réactions face à un proche qui ne se soigne pas.
}

\author{
Alexis Ferrand \\ Ancien professeur de sociologie à l’Université de Lille. \\ Revue Française des Affaires Sociales, 2019, № $1: 39-53$ \\ NB : Ce fichier n’a pas la pagination éditée.
}

\begin{abstract}
Résumé
Les aidants profanes sont des acteurs importants de la prise en charge des malades. Cet article présente une démarche originale qui examine les trajectoires d'engagement d'un acteur dans l'aide en matière de santé. D'abord comment peut-il percevoir parmi ses relations une personne qui s'occupe mal de sa santé ou même qui ne s'aperçoit pas qu'elle a un problème de santé ? Ensuite envisage-t-il d'intervenir, et comment, auprès de cette personne ?

Le cadre d'analyse est celui de l'interactionnisme symbolique et la méthodologie est dérivée de l'analyse des réseaux personnels. Une pré-enquête réalisée en milieu aisé ( $\mathrm{N}=140)$ montre qu'on peut obtenir des informations de base sur la surveillance relationnelle de la santé. Elle interdit évidemment toute généralisation mais suggère des interrogations, notamment sur la réticence de certains acteurs à intervenir auprès d'une personne dont ils perçoivent cependant qu'elle a un problème de santé.
\end{abstract}

\section{Introduction}

Les interventions des aidants familiaux, ou plus généralement des aidants à la fois profanes et bénévoles, sont de mieux en mieux prises en compte dans les réflexions et les analyses sur le système de soins. La proportion grandissante de personnes très âgées, grandes demandeuses de soins, dans une conjoncture économique atone et de déficit de tous les comptes sociaux, a attiré l'attention sur les aidants qui ne coûtent rien tout en procurant des services considérables. Alors que les analyses s'intéressaient auparavant d'un coté aux comportements des individus (dans un esprit de prévention des risques) et de l'autre aux services de santé, on cherche à présent à explorer un nouvel objet : le réseau des aidants profanes qui contribuent à la prise en charge des personnes. Fin 2015 la Direction de la recherche et des études économiques (DREES) et l'Institut national de la statistique et des études économiques (INSEE) ont ainsi terminé deux volets d'un grand programme d'analyse des «Capacités, aides et ressources des seniors » (CARE, 2015, 2018) qui décrit précisément les relations d'aide qui interviennent en faveur d'un individu ${ }^{1}$. Cette importante enquête s'inscrit dans la tradition, déjà longue dans la littérature anglo-saxonne, d'études des fonctions des réseaux de relations personnelles dans la protection de la santé des individus et dans la prise en charge de leurs maladies (Berkman et Glass, 2000 ; Ferlander, 2007).

Dans cet article nous allons pareillement nous intéresser aux relations d'un individu. La question habituelle est de savoir quels membres de son réseau peuvent l'aider. Nous renversons la question pour nous demander si un acteur quelconque peut s'apercevoir qu'il a

\footnotetext{
110.643 séniors enquêtés, avec, pour chacun, une description rapide de toutes ses relations d'aide non professionnelles et 6251 aidants directement enquêtés pour décrire qui ils sont et quelles conséquences sociales et sanitaires implique leur fonction d'aidant (Besnard, Brunel et Carrère, 2015).
} 
dans son réseau quelqu'un dont la santé pourrait exiger qu'il intervienne. Nous utilisons le terme «d'acteur » parce que la question est aussi de savoir s'il va intervenir, agir. Et les relations dont il sera question comportent au minimum les interactions et les échanges face à face qui permettent à l'acteur de voir physiquement une personne.

Nous envisageons la période qui précède le déclenchement de l’aide, période où cet acteur va, ou non, percevoir qu'un membre de son réseau a un problème de santé, de quel type de problème il s'agit, et envisager éventuellement d'intervenir. Cette démarche a deux objectifs : d'une part suggérer que certains acteurs, pas tous, peuvent surveiller la santé de leurs proches et, parfois, devenir des aidants; d'autre part un objectif méthodologique : vérifier si un module de quelques questions (destiné à être intégré dans une enquête nationale) peut décrire à grand traits ces relations de surveillance. Mais les moyens sont limités : la pré-enquête réalisée dont les résultats sont présentés ici porte sur un échantillon étroit destiné simplement à ouvrir une discussion.

Dans une première partie, nous rappelons que décréter qu'une personne a un problème de santé ou dire qu'elle est malade implique parfois des controverses et des interprétations divergentes entre profanes mais aussi avec des soignants. Nous indiquons ensuite les catégories d'analyse utilisées dans l'approche relationnelle que nous avons esquissée. Dans une seconde partie nous utilisons une petite pré-enquête pour examiner quelques dimensions du processus de surveillance relationnelle de la santé. Nous insistons, pour conclure, sur l’importance du contrôle micro-social des comportements de santé.

\section{Comment un acteur peut-il juger qu'une personne s'occupe mal de sa santé?}

\subsection{La construction sociale problématique du rôle de malade}

Comprendre notre corps est complexe. On se demande si certaines manifestations corporelles devraient être interprétées comme des symptômes de quelque chose, symptômes d'un petit trouble passager ou, au contraire, symptômes d'une vraie maladie. Habituellement on mobilise l'avis de différents conseillers, d'abord profanes, puis professionnels, pour se faire une opinion. Eliot Freidson (1960) a très bien décrit ce parcours. Le réseau personnel a ainsi une fonction cognitive pour l'individu : certains membres donnent des conseils, aident à mettre un nom médical sur des troubles ressentis, ou permettent de considérer que tout va bien. Mais il a aussi une fonction normative. Quand des membres de son réseau profane, ainsi que des professionnels consultés, reconnaissent une personne comme « malade », des normes sociales lui accordent des droits nouveaux (cesser le travail, délaisser des tâches habituelles, se faire aider pour certaines activités, etc.) et des obligations nouvelles (notamment de se soigner, de prendre des médicaments prescrits par un médecin, de faire des examens biologiques, etc.). Ces droits et ces obligations constituent le "rôle de malade ", c'est-à-dire des normes nouvelles qui guident les relations entre la personne et différents membres, professionnels et profanes, de son environnement social. Mais ce schéma est passablement idéal. L’interactionnisme symbolique a mis en évidence que les changements de rôles qui modifient les relations entre l'individu et ses différents partenaires ne fonctionnent pas de manière harmonieuse (Baszanger, 1992 ; Dodier, 1983). Entre la personne concernée, son employeur, sa famille, des proches consultés, des professionnels de santé, les interprétations de la situation sont souvent différentes parce qu'elles mobilisent des références et des normes divergentes (Freidson, 1970). Les critères pris en compte par les uns ou les autres pour décider qu'une personne peut être définie comme "malade » sont hétérogènes, et entraînent parfois des conflits. Donc, pour un acteur, simplement percevoir un proche comme 
« malade », ou comme ayant « un problème de santé » est un fait qui est loin d'être anodin et qui peut l'engager dans un conflit d'interprétation avec d'autres proches de cette personne.

Dans quels types de relations interpersonnelles un acteur a-t-il des chances (des risques !) de se sentir concerné par la santé d'un membre de son réseau ? On sait que certaines relations veillent à ce que l'individu se conforme à ses obligations et l'aident à rester en bonne santé (Parizot, Chauvin, Firdion, et al., 2004). Dans cet esprit, nous avons analysé comment les confidents en matière affective et sexuelle pouvaient aider l'individu à adopter des normes préventives face au VIH (Ferrand et Mounier, 1998). On avait également envisagé comment un réseau de relations interpersonnelles pouvait surveiller les comportements sexuels de ses membres et, au besoin, sanctionner des pratiques à risque de transmission du VIH (Ferrand et Snijders, 1997). Or seules certaines relations comportent des interactions qui permettent d'identifier des problèmes de santé émergents et il faut donc savoir lesquelles.

Enfin, il y a des personnes qui, pour des raisons très diversifiées, refusent de se déclarer comme malades, d'admettre le diagnostic officiel d'un médecin ou les suggestions profanes de leur entourage. Elles refusent d'assumer les obligations d'un malade (prendre les précautions requises, accepter les traitements prescrits, etc.) et elles refusent aussi parfois les droits propres à ce statut ${ }^{2}$. Elles ne se soignent pas, ou ne se soignent pas selon les normes de la médecine officielle.

\subsection{Les catégories d'analyse}

Le cadre d'analyse qui a présidé à l'élaboration du test méthodologique présenté ici a tenu compte de l'objectif visant à introduire un nombre limité d'items dans des questionnaires destinés à des sondages nationaux. Notamment, il n'est pas possible d'explorer les contenus des divergences normatives qui ont été évoquées plus haut. C’est donc la doxa médicale officielle qui fait référence que ce soit dans le langage du questionnaire, pour des raisons de rapidité de compréhension, ou dans le choix des catégories utilisées.

Des personnes qui savent qu'elles ont un problème de santé, mais ne font pas ce qui est médicalement recommandé, ni en terme de vie quotidienne, ni en terme de traitement, sont dans des situations de "renoncement aux soins ». Philippe Warin $(2010,2011)$ en a montré les raisons variées, et souvent contraintes par des obstacles économiques ou sociaux. Pour notre part, nous appuyant sur le langage courant à forte tonalité moralisatrice qui est celui des acteurs, nous dirons que ces personnes négligent leur santé, qu'elles sont donc « négligentes ».

Cependant, certaines personnes sont dans des situations encore plus critiques car elles ne s’aperçoivent même pas qu'elles ont un problème de santé. Nous dirons alors qu'elles sont en situation de « déni d'un problème de santé ». Nous employons le terme de "déni » car nous postulons qu'il y a souvent une part non consciente dans l'occultation de tout signal d'un mal être.

Ces deux types de comportements posent de graves problèmes de santé publique et des programmes de recherche (Boisguerin, 2012) ont examiné les processus qui font :

- que des personnes qui se savent malades ne se soignent pas ;

- que des personnes n'ont pas conscience d'être malades et d'avoir besoin de soins.

Pour comprendre pourquoi des personnes qui se savent malades ne se soignent pas, il est possible d'enquêter auprès des populations particulières dont on sait que la prévalence du

\footnotetext{
${ }^{2}$ Refus de prendre un congé de maladie, par exemple, en anticipant des risques professionnels (Barnay, Favrot et Pollak, 2015).
} 
renoncement aux soins est importante ${ }^{3}$. En revanche, comprendre pourquoi certaines personnes n'ont pas conscience d'être malades pose des problèmes d'enquête redoutables car il est complexe de chercher à interroger un individu sur des faits dont, par hypothèse, il n'a pas conscience.

Sans répondre strictement à cette difficulté, l'analyse que nous présentons ici est nouvelle car elle déplace la question : au lieu de chercher si l'individu perçoit ses propres problèmes, elle examine si un proche de son entourage peut percevoir les problèmes de santé négligés ou refoulés par ce proche.

Dans cette approche, nous mobilisons un paradigme relationnel: l'analyse concerne les relations qui permettent, ou qui ne permettent pas, à un acteur de détecter dans son entourage une personne négligente et qui favorisent une intervention auprès d'elle. Les relations interpersonnelles sont soumises à des régulations complexes qui concourent à définir les interactions qu'elles peuvent comporter et donc les fonctions qu'elles peuvent remplir : soutien émotionnel, aides cognitives ou matérielles, contrôle et sanction, etc. (Ferrand, 2007). Ici nous retenons un modèle classique selon lequel les relations sont orientées par des normes sociales qui, au-delà des variantes propres à différents milieux sociaux, se cristallisent dans des rôles typiques :

a) Les rôles des femmes ${ }^{4}$ et les rôles des hommes : on sait que les hommes et les femmes n’ont pas les mêmes pratiques de santé. Et ces pratiques sont largement constituées par des relations entre des femmes et des hommes, les premières se voyant souvent assigner la prise en charge des seconds (Cresson, 1995, 2001). Il convient donc d'examiner les quatre types de combinaison des genres entre un acteur et une personne de son entourage.

b) Les contextes : les relations sont inscrites dans des contextes sociaux et orientées par des normes de rôle différentes selon qu'il s'agit de relations familiales, entre collègues, entre camarades de loisirs, ou autres. Ces rôles relationnels permettent plus ou moins aux uns de laisser transparaître des problèmes de santé et aux autres de les percevoir et d'intervenir.

Enfin, il faut préciser comment un acteur peut être concerné par la santé de personnes dans son entourage. Nous parlerons de «la surveillance relationnelle de la santé » pour désigner un processus, qui peut être décomposé en trois moments :

a) En premier lieu, un acteur perçoit dans son entourage une personne qui présente certaines anomalies dans le registre des signes corporels, des attitudes et des comportements de santé. b) En second lieu, l'acteur interprète ces signes, ces comportements, ou bien des propos de cette personne et va lui attribuer un problème de santé particulier. Il jugera alors si cette personne tient correctement son rôle de malade, c'est-à-dire fait ce que peut prescrire la médecine.

c) En troisième lieu, l'acteur peut (ou non) tenter d'intervenir auprès de la personne s'il juge qu'elle ne fait pas ce qui serait nécessaire ${ }^{5}$.

Ces processus, qui sont en quelque sorte en amont du care, ont été peu étudiés dans des grandes enquêtes en santé publique et nous voulions savoir si un module de quelques questions spécifiques permettrait d’en proposer un aperçu. Nous avons donc réalisé une pré-

\footnotetext{
${ }^{3}$ Bien que cette méthode puisse poser des problèmes d'observation comme ceux que Renaud Legal et Augustin Vicard (2015) ont mis en évidence à propos des raisons financières.

${ }^{4}$ On admettra que, selon différents milieux sociaux, «le » rôle des femmes peut varier, et qu'il existe donc « des » rôles des femmes, pareillement pour les hommes. Mais ces rôles sont cependant articulés autour de quelques « attributs fondamentaux » typiques (Nadel, 1957) que les enquêtes détectent.

${ }^{5}$ Il est évident que si on disposait de 30 questions, il faudrait introduire la possibilité d'un désaccord entre l'acteur et les prescriptions médicales imposées à la personne.
} 
enquête dont les résultats sont utilisés ici pour illustrer le propos et permettre de formuler quelques interrogations.

\section{Une pré-enquête sur la surveillance relationnelle de la santé}

\section{La pré-enquête : « La santé : un souci de l'entourage"}

Cette pré-enquête visait à tester un nombre limité de questions pouvant être ultérieurement incluses dans une enquête auprès d'un échantillon représentatif. Ces questions devaient permettre d'identifier quels enquêtés perçoivent ou non parmi leurs proches une personne qui néglige sa santé, ou qui refuse de percevoir ses problèmes de santé, et comment ces enquêtés interviennent (ou non) auprès de cette personne. Des caractéristiques socio-démographiques très sommaires ont été relevées ici, en supposant qu'elles seraient largement développées ailleurs dans une enquête plus large. Elles ont limité les analyses possibles.

Le questionnaire a été diffusé par mail dans des réseaux amicaux sous forme informatisée. 140 réponses ont été obtenues : 64\% de femmes et 36\% d'hommes. $81 \%$ de profession libérales et cadres supérieurs. Cette concentration sur un groupe social est un effet prévisible de la diffusion «boule de neige » du questionnaire à partir de trois sociologues académiques, ce qui limite la généralisation des résultats. On trouvera une présentation détaillée de cette pré-enquête dans Ferrand A. (2013, téléchargeable sur Halshs).

\subsection{Que remarquent les profanes dans leur entourage ?}

La question initiale était formulée dans les termes suivants : "Parmi les adultes que vous connaissez, il y a sans doute des personnes qui s'occupent beaucoup de leur santé, et d'autres qui n'y font pas du tout attention. Vous pouvez penser à vos collègues, à des membres de votre famille, à vos amis, ou à d'autres connaissances. Parmi ces adultes que vous connaissez avez-vous remarqué une personne qui ne s'occupe pas de sa santé d'une des manières suivantes :

Tableau 1 : Remarquer une personne qui ne s'occupe pas de sa santé

\begin{tabular}{|l|c|c|}
\hline $\begin{array}{l}\text { Parmi ces adultes que vous connaissez avez-vous remarqué une personne } \\
\text { qui ne s'occupe pas de sa santé d'une des manières suivantes }\end{array}$ & $\mathrm{N}$ & $\%$ \\
\hline Je n'en connais aucune qui ne s'occupe pas de sa santé. & 59 & $42 \%$ \\
\hline $\begin{array}{l}\text { J'en connais au moins une qui ne s'occupe pas des problèmes de santé } \\
\text { qu'elle connaît. }\end{array}$ & 69 & $49 \%$ \\
\hline J'en connais une qui ne s'aperçoit pas des problèmes de santé qu'elle a. & 12 & $9 \%$ \\
\hline Tou·te·s répondant·e·s & 140 & $100 \%$ \\
\hline
\end{tabular}

Note : Le second item suppose que l'enquêté-e perçoit une personne qui «renonce aux soins », le troisième une personne en situation de «déni d'un problème de santé. »

Au total, 58\% des répondants-tes ont détecté parmi leurs proches au moins une personne qui ne s'occupe pas de sa santé. Ceci indique que la surveillance des comportements de santé des proches existe, et qu'elle est suffisamment fine, discriminante, pour détecter un proche qui ne 
se soigne pas ${ }^{6}$. De manière encore plus remarquable, 12 acteurs (9\%) disent percevoir une personne «qui ne s’aperçoit pas des problème de santé qu'elle a ». Au sein du processus de surveillance, dont on recueille ici l'aveu, ce résultat montrerait l'existence étonnante d'une capacité de diagnostic profane par un proche d'un problème de santé ignoré par la personne. Il s'agirait alors d'une puissante capacité de perception et d'interprétation des signes corporels et des propos de la personne.

Des hommes et des femmes.

Il est admis que les femmes sont plus attentives à la santé de leur entourage que les hommes. Cependant, dans la petite population étudiée, les femmes sont aussi nombreuses que les hommes à percevoir une personne négligente dans leur réseau (59\% et 55\%). Les hommes seraient donc aussi attentifs que les femmes à la santé de leurs proches, ce qui est inattendu. Mais les femmes citent deux fois plus souvent un homme négligent qu'une femme négligente (35 hommes contre 17 femmes), alors que les hommes citent les unes et les autres de manière plus équilibrée (12 femmes contre 15 hommes).

Il s'agit d'une asymétrie de détection du problème, car, sauf biais d'enquête très particulier, les hommes et les femmes qui ont répondu fréquentent les mêmes types d'hommes et de femmes. Si « les femmes sont plus attentives à leur santé que les hommes » (Aliaga, 2002), elles sont cependant plus nombreuses que les hommes à renoncer à des soins pour raisons financières (19,8\% contre 13,0\%. Irdes, 2008). On peut formuler l'hypothèse que la propension des femmes à repérer dans leur entourage moins de femmes que d'hommes qui ne s'occupent pas de leur santé peut être due en partie aux normes de rôle féminin qui suggèrent qu'elles prennent davantage soin d'elles mêmes, mais aussi à l'obligation qui leur est faite d'être disponibles pour faire face aux problèmes des proches, et donc à l'obligation de dissimuler leurs propres problèmes aux yeux de leur entourage. Comme le notait Marcel Druhle (1996), « les rôles d'épouse et de mère ne sont pas de ceux qui portent à s’écouter».

Exclusivement la sphère familiale

Aux enquêtés qui disaient avoir repéré quelqu'un qui s'occupe mal, ou qui refuse de voir un problème de santé $(n=81)$, le questionnaire demandait "Comment connaissez-vous cette personne ? » et permettait plusieurs réponses dans une série de propositions sur des relations typiques (par exemple, " collègue ", "famille proche, ou vit à la maison », ou " partenaire sexuel, époux, épouse », etc.). Conformément à ce qu’on connaît par ailleurs, lorsqu'il s’agit d'enjeux personnels, les relations qui peuvent les évoquer appartiennent massivement (deux tiers) à la sphère privée de la famille et des partenaires sexuels. Il ne s'agit ni de collègues, ni de camarades de loisirs ou d'association, ni de simples amis ou connaissances. Ce n'est pas parce que ces relations sont moins nombreuses que les relations familiales, mais bien parce que ce sont les rôles particuliers de ces relations qui prescrivent plus fortement à l'acteur de se soucier de l'état de santé de ces personnes.

\subsection{Les problèmes de santé qui peuvent être perçus par l'entourage}

Le questionnaire demandait ensuite à ces 81 répondants-tes de dire, parmi 11 "problèmes de santé », lesquels concernaient la personne qu’ils avaient repérée ${ }^{7}$. Les répondants-tes ont attribué en moyenne 1,8 problèmes à chaque personne qui renonce aux soins et 1,3 à celle qui ne reconnait pas sa maladie. Ce qui suggère que les enquêté-e-s n'ont pas une vague appréhension de la santé de la personne envisagée, mais qu'ils sont au contraire capables

\footnotetext{
${ }^{6}$ Il faut bien noter que, même dans une enquête auprès d’un échantillon national représentatif, la fréquence de la perception des personnes négligentes n’indiquerait en rien la prévalence réelle des cas de négligence.

Sur une liste dérivée de l'enquête IRDES ESPS 2008.
} 
d'attribuer non seulement un, mais aussi plusieurs, problèmes de santé différenciés à cette personne. Il est en plus possible que les enquêté-e-s ne mentionnent pas d'autres problèmes dont cette personne s'occuperait. Cette attention peut être spécifique à la classe moyenne des enquêté-e-s. Mais la banalisation dans l'ensemble de la population, notamment via le web, des informations sur les maladies, pourrait en faire une capacité largement répandue.

Que perçoivent les enquêté-e-s ? Les problèmes d'addictions sont cités par un tiers (35\%), et les problèmes psychologiques par un quart (23\%), des répondants-tes. Mais cette prédominance n'empêche pas la mention d'autres problèmes par $10 \%$ à $20 \%$ des enquêtés-es. Et la très faible citation des problèmes de cancer est due au fait qu'il est rare qu'une personne ne s'occupe pas de son cancer, et encore plus rare qu'un tiers sache que la personne a un cancer dont elle ignorerait l'existence. Je n'insiste pas sur ces points pour examiner la suite qui me semble soulever plus de questions sociologiques.

\subsection{Intervenir ou garder ses distances}

Une enquête large confirmerait sûrement que la surveillance relationnelle de la santé est bien une préoccupation et une interaction fréquentes dans les relations entre proches. Il existe des "veilleurs profanes" qui sont des acteurs décisifs pour découvrir les personnes qui s'occupent mal de leur santé. Mais que font-ils ensuite ? Après avoir perçu une personne « qui ne s'occupe pas des problèmes de santé qu'elle connaît », après avoir identifié son (ses..) problème de santé, que décident-ils, vont-ils intervenir, et comment ? Finalement découvrir la négligence d'un proche, est-ce le premier pas vers l'entrée dans le rôle d'aidant profane ?

Pour explorer ces questions, pour savoir si, et de quelle manière, l'acteur intervient vis-à-vis de la personne négligente, on a posé la question suivante : "Qu'avez-vous fait vis-à-vis d'elle au cours des 6 derniers mois ?"

Tableau 2. Les types d'intervention auprès des personnes négligentes

\begin{tabular}{|l|c|c|}
\hline Qu'avez-vous fait vis-à-vis d'elle au cours des 6 derniers mois ? & $\mathrm{N}$ & $\%$ \\
\hline Vous lui avez dit que vous vous faisiez du souci pour sa santé. & 43 & $53 \%$ \\
\hline Vous lui avez donné des conseils précis pour sa santé. & 29 & $36 \%$ \\
\hline Vous avez parlé avec quelqu'un qui peut lui donner des conseils. & 23 & $28 \%$ \\
\hline Vous n'avez rien dit parce que cela ne servirait à rien. & 9 & $11 \%$ \\
\hline Vous n'avez rien dit parce que c'est son affaire. & 5 & $6 \%$ \\
\hline Nombre d'interventions citées par 81 répondant·e·s $(m=1,3)$ & 109 & \\
\hline
\end{tabular}

Note : Sous-ensembles des 81 enquêté·e·s ayant cité une personne négligente.

Multiréponses, donc le total des citations (109) est supérieur au nombre d'acteurs (81) et le total des pourcentages est supérieur à 100 .

La première proposition « vous lui avez dit que vous vous faisiez du souci pour sa santé » évoque une intervention que l'on peut qualifier de peu coûteuse en termes relationnels. L'item a été formulé dans cet esprit de façon à regrouper l'ensemble des discussions où le " souci » est une marque d'attention et de sympathie dans le jeu de la sociabilité. Mais dire à une personne qu'on «se fait du souci pour sa santé » peut parfois impliquer un reproche : «si tu veux me faire plaisir, si tu veux que je ne me fasse plus de souci, occupes-toi mieux de ton problème ». Ce type d'intervention engage peu l'acteur vis-à-vis de la personne, elle peut être considérée comme relativement facile, elle est en tout cas est la plus fréquente car citée par $53 \%$ des acteurs. 
La seconde intervention proposée "vous lui avez donné des conseils précis pour sa santé » décrit un engagement nettement plus fort de l'acteur car il doit faire preuve d'une connaissance approfondie du problème de la personne ainsi que des soins qui conviennent. De plus, l'acteur prend presque une responsabilité de "prescripteur profane " en donnant des conseils précis. Or, parmi les acteurs qui ont détecté une personne négligente, seulement quatre sur dix (36\%) ont déclaré cette intervention forte, et il faut se demander ce que peuvent être les interventions ultérieures de l'acteur. Il faudrait, dans une enquête large, examiner dans quelle mesure cet engagement serait un préliminaire à l'entrée dans un rôle plus affirmé de surveillant et/ou d'aidant.

Plus surprenant, trois acteurs sur dix utilisent un moyen d'intervention détourné en "parlant à quelqu'un qui pourrait donner des conseils » à la personne (28\%). Ce résultat est tout à fait important parce qu'il indique la possibilité d'une intervention qui n'est plus seulement interpersonnelle (l'acteur - la personne : intervention dyadique) mais qui implique un tiers, et devient donc triadique (l'acteur - un tiers - la personne). Il faut bien percevoir que cette intervention fonctionne à un niveau différent : les réseaux personnels des deux acteurs sont alors en cause, et non plus une relation isolée entre eux. En effet cette intervention est conditionnée par l'existence de différentes relations qui seraient communes aux réseaux personnels de l'acteur et de la personne et l'analyse devrait donc pouvoir porter sur «des structures relationnelles de surveillance de la santé ».

Enfin, un acteur sur dix « n'a rien dit parce que ca ne servirait à rien » (11\%) et un sur vingt «n'a rien dit parce que c'est son affaire " $(6 \%)$. Ne rien dire, c'est ne même pas s'engager dans l'intervention faible que nous avons évoquée en premier. L’absence d'intervention est deux fois plus souvent justifiée par une absence d'influence, donc d'efficacité de l'intervention, que par une absence de légitimité. Ce fait, s'il est confirmé par une enquête large, est également crucial. Il indiquerait bien, a contrario, qu'il est légitime de se préoccuper de la santé de ses proches et, éventuellement, d'intervenir, mais sans illusion, en étant conscient des limites de notre relation à l'autre.

Finalement et pratiquement, un grande nombre d'enquêtés ne s'impliquent pas fortement. Ils sont nombreux à garder leur distance à l'égard d'un proche qui ne s'occupe pas bien de sa santé : soit en manifestant seulement un souci superficiel, soit en faisant appel à un tiers, soit en reconnaissant leur impuissance. Mais la norme reste de s’intéresser à la question : la santé d'autrui n'est pas seulement son affaire, mais aussi la mienne.

\section{Conclusion}

Les résultats que nous venons de commenter ne démontrent évidemment rien concernant la population française dans son ensemble. Ils permettent cependant, d'une part, de penser qu'un nombre limité de questions, dans une enquête large, fournirait un aperçu intéressant des processus de surveillance relationnelle de la santé. Ils sont alors un acquis méthodologique. Ils permettent, d'autre part, de formuler quelques questions supplémentaires.

Les modèles d'analyse individualistes s'intéressent aux moyens, aux motivations et aux raisons que peut avoir un individu de se préoccuper de sa santé et de se soigner. Et ils mettent aussi " en cause " des déterminants collectifs qui pèsent sur lui : disponibilité de ressources pratiques et cognitives, contexte culturel, etc (Goldberg, 2002). On utilise ici un modèle complémentaire qui considère l'individu comme inséré dans un réseau de relations interpersonnelles dont les aidants profanes sont les membres les plus actifs si sa santé est atteinte. Mais ces aides importantes ne sont ni les plus générales, ni les seules. De manière plus courante, certains membres de son réseau surveillent sa santé et, parfois, tentent d’intervenir. Nous avons présenté et illustré ce processus, situé en quelque sorte en amont du 
rôle d'aidant. En quoi est-il important ? En ce qu'il est une des formes du contrôle social des comportements de santé. Outre les facteurs sociaux et environnementaux exogènes qui pèsent sur ses marges d'action, l'individu n'est pas seul responsable de sa santé : son réseau de relations personnelles peut contribuer à le maintenir «dans le droit chemin ». Mais nous connaissons mal ce processus et seule une enquête large permettrait de préciser s'il est fréquent, et comment il fonctionne: quels types de relations permettent de percevoir que quelqu'un ne s'occupe pas de sa propre santé, et quelles interventions ces relations autorisentelles pour y remédier. Quelles interventions permettent-elles pratiquement, mais surtout quelles interventions permettent-elles normativement: jusqu'à quel point une relation personnelle peut demander (imposer ?) à un de ses partenaires de s'occuper de « sa » santé.

Quelques questions mériteraient particulièrement l'attention. D'une part, il faudrait analyser la distribution de ces processus dans différents contextes sociaux, en entendant ceux-ci à la fois comme déterminants macro-sociaux (par exemple appartenances à différentes CPS) et comme déterminants micro-sociaux (de quels types de relations personnelles l'individu dispose-t-il ? Quelle dépendance affective peut l'attacher particulièrement à quelqu'un ? Quel proche aurait le pouvoir de le contraindre ? etc.).

D'autre part, il conviendrait de comprendre pourquoi des acteurs ne s'engagent que faiblement. Et notamment comment un acteur ayant un type de relation peu légitimé à intervenir sur la santé de son partenaire tente de mobiliser un autre type de relation plus légitime ou plus efficace : le collègue d'une personne négligente peut parler au frère de celleci, qui serait plus influent pour donner des conseils (ce qui constitue une «triadisation » du contrôle social qui met alors en cause les connaissances communes aux deux). Ceci peut introduire une interrogation plus générale : comment parmi les connaissances d'une personne, l'une d'elles est incitée à intervenir à cause de la pression morale des autres et/ou avec la reconnaissance et les remerciements des autres (Petite, 2005), ce qui est une variante d'un problème bien identifié par James Coleman (1990) : comment remercier les acteurs profanes qui s'occupent de sanctionner les individus qui ne contribuent pas au bien commun.

D’un point de vue plus théorique, il convient de remarquer que l'explication par les normes de rôles qui servent de références dans les ajustements des interactions n'est qu'un premier apport à la connaissance. Cette interprétation convient pour les données limitées qu'offre un module particulier inséré dans une enquête nationale de santé publique. Il serait cependant possible, avec des investigations spécifiques, d'explorer d'autres explications. D'une part en mobilisant une sociologie de l'acteur rationnel : un acteur peut avoir de "bonnes raisons » (Boudon, 2002) de vouloir qu'une personne de son entourage se porte bien parce que d'une manière directe ou indirecte c'est dans son intérêt (au sens large). D'autre part on peut invoquer une sociologie de l'échange. Se soucier de la santé d'une personne peut consister à s'acquitter d'une dette et les variantes sont très nombreuses (Ekeh, 1974 : échange restreint, chaîne d’échanges, échange généralisé).

Pour terminer, il apparaît essentiel de vérifier, lors d'une enquête large, si la surveillance et l'intervention relationnelles sont, comme elles sont apparues ici, a priori légitimes. Il peut il y avoir un débat axiologique : ces interventions peuvent être jugées très positives si la santé est érigée en valeur prééminente, mais le jugement change si la liberté et la responsabilité individuelles sont posées en valeurs prééminentes. 


\section{Références bibliographiques}

Barnay T., Favrot J., et Pollak C. (2015), « Les arrêts maladie de longue durée pénalisent les trajectoires professionnelles », Études et Résultats, n938, Paris, Drees.

http://www.drees.sante.gouv.fr/IMG/xls/er938.xls

Baszanger I. (ed.), (1992), La trame de la négociation: sociologie qualitative et interactionnisme, Paris, L'Harmattan, 1992

Besnard X., Brunel M., et Carrère A. (2015), Care News, $n^{\circ} 2$.

http://www.drees.sante.gouv.fr/IMG/pdf/carenews2.pdf

Berkman L.F., Glass T. (2000), "Social Integration, Social Networks, Social Support and Health”, in Berkman L.F. et Kawachi I., (dir.), Social Epidemiology, Oxford, University Press : 137-173. http://www.hsph.harvard.edu/faculty/LisaBerkman.html

Boisguerin B. (dir.) (2012), « Renoncement aux soins. Actes du colloque du 22 novembre 2011 », Paris, DREES, Collection études et statistiques.

http://www.drees.sante.gouv.fr/IMG/pdf/actes_renoncement_soins_2012.pdf

Boudon R. (2002), Raisons, bonnes raisons, Paris, PUF

CARE, (2015) «Capacités, Aides et Ressources des Seniors », Paris, DREES \& INSEE.

Volet seniors : http://www.drees.sante.gouv.fr/IMG/pdf/care_plaquette_seniors.pdf

Volet aidants : http://www.drees.sante.gouv.fr/IMG/pdf/care_plaquette_aidants.pdf

CARE (2018) https://drees.solidarites-sante.gouv.fr/IMG/pdf/27032018_support_vf.pdf

Coleman J.S. (1990), Foundations of social theory, Chicago, University of Chicago Press

Cresson G. (2001), " Les soins profanes et la division du travail entre hommes et femmes », in Aïach P., Cébe D., Cresson G., et al., Femmes et hommes dans le champ de la santé.

Approches sociologiques, Rennes, ENSP : 303-328.

Cresson G., (1995) Le travail domestique de santé, analyse sociologique, Paris, L’Harmattan.

Dodier N., (1983), « La maladie et le lieu de travail », Revue Française de Sociologie, XXIV

Drulhe M., (1996) Santé et société. Le façonnement sociétal de la santé. Paris, PUF.

Ekeh P., (1974) Social exchange theory: the two traditions, Cambridge, Harvard University

Press.

Ferlander S., (2007) “The Importance of Different Forms of Social Capital for health”, Acta

Sociologica, Vol. 50, No. 2 : 115-128. http://www.jstor.org/stable/20459987

Ferrand A., (2013) La santé : un souci de l'entourage, Lille, CNRS Clersé.

https://halshs.archives-ouvertes.fr/halshs-00790384

Ferrand A., (2007) Confidents, Une analyse structurale de réseaux sociaux, Paris,

L'Harmattan.

Ferrand A., Mounier L.,(1998) «L’influence des réseaux de confidence sur les relations

sexuelles », in Bajos N., Bozon M., Ferrand A., et al. (dir.) La sexualité aux temps du Sida ,

Paris, PUF : 255-304. http://halshs.archives-ouvertes.fr/halshs-00253901/fr/

Ferrand A., Snijders T., (1997) "Social networks and normative tensions", in Van

Campenhoudt L., Cohen M., Guizzardi G., et al. (dir.), Sexual interactions and HIV risk : new

conceptual perspectives in European research, London, Taylor \& Francis : 6-21.

http://halshs.archives-ouvertes.fr/halshs-00264362/fr/

Freidson E., (1970) Profession of Medicine, Traduction française La profession médicale,

Paris, Payot, 1984.

Freidson E., (1960) “Client control and medical practice.”, American Journal of Sociology, 65 : 374-382. Traduction Française dans Médecine, maladie et société, C.Herzlich (dir.) Paris,

Mouton, 1970.

Goldberg M., Melchior M., Leclerc A., et al., (2002), « Les déterminants sociaux de la santé : apports récents de l'épidémiologie sociale et des sciences sociales de la santé. », Sciences sociales et santé, Volume $20, \mathrm{n}^{\circ} 4: 75-128$ 
HAS, (2010) Maladie d'Alzheimer et maladies apparentées : suivi médical des aidants naturels, Paris, HAS.

http://www.has-sante.fr/portail/upload/docs/application/pdf/201112/recommandation_maladie_dalzheimer_-_suivi_medical_des_aidants_naturels.pdf IRDES, (2008) Enquête sur la santé et la protection sociale, http://www.irdes.fr/Publications/Rapports2010/rap1800.pdf

Legal R., Vicard A. (2015), « Renoncement aux soins pour raisons financières », Dossiers Solidarité et Santé, n 66, Paris, DREES

Nadel S.F., (1957) The theory of social structure. London, Cohen et West, Traduction Française, La théorie de la structure sociale, Paris, Editions de Minuit, 1970.

Parizot I., Chauvin P., Firdion J.M. et al. (2004), Santé, inégalités et ruptures sociales dans les zones urbaines sensibles d'Ile-de-France, Paris, Observatoire national de la pauvreté et de l'exclusion sociale.

https://travail-emploi.gouv.fr/IMG/pdf/Travaux2003-2004.2.2.2.sante_zus_idf.Parizot.pdf Petite S., (2005) Les règles de l'entraide. Sociologie d'une pratique sociale, Rennes, PUR Warin P., (2010) Le non-recours : définition et typologies, Document de travail $\mathrm{N}^{\circ} 1$, Grenoble, ODENORE. https://odenore.msh-alpes.fr/documents/odenorewp1.pdf Warin P., (2011), « Renoncement à des soins et précarité », communication au colloque Le Renoncement aux soins, Novembre 2011, Paris, DREES, Col. Etudes et Statistiques http://www.drees.sante.gouv.fr/IMG/pdf/actes_renoncement_soins_2012.pdf 